\title{
How Safe Is Your Neighborhood? Iranian Women's Perception of Safety and Security
}

\section{Shideh Shakouri AsI}

\author{
Ph.D. in Urban planning, Department of Art and Architecture, Tehran Science and Research Branch, \\ Islamic Azad University, Tehran, Iran \\ Corresponding Author Email: shdhshakouri@gmail.com
}

\author{
Azadeh Lak \\ Ph.D. in Urban Design (Tehran) Assistant Professor, Faculty of Architecture \& Urbanism, Shahid Beheshti \\ Email: University.A_lak@sbu.ac.ir
}

\author{
Doi:10.5901/mjss.2017.v8n1p419
}

\section{Abstract}

Safety and security are considered as basic human needs and fundamental rights. Women, who are among the most vulnerable groups, need more safety and security in neighborhoods. Using a conceptual model, this study explored factors contributing to women's perceptions of safety and security in their neighborhood. In the context of the North Salsabil neighborhood of Tehran (Iran's capital city), a combination of both qualitative and quantitative methods was applied. The qualitative method was based on the responses of 20 women to semi-structured interview questions; the responses were analyzed applying content analysis. In the quantitative phase, stratified random sampling was used to contact the female participants $(N=200)$ aged 18 to 65 years old. The measurement instrument used was a questionnaire based on conceptual model indices. The results revealed that women's perception of security in the neighborhood was associated with three main factors: individual characteristics, local community features, and built environment characteristics.

Keywords: Safety, security, women's perception of security, the North Salsabil neighborhood.

\section{Introduction}

Built settlements and neighborhoods are important contributors to individual's social and psychological well-being. The built environment could add to, or subtract from, individuals' sense of security and safety (Nasar and Jones, 1997). Women's safety is important as it is a factor in creating healthy and vibrant neighborhoods. Women face many risks and dangers in urban areas, such as theft, purse snatchers (mugging), getting lost, or they may even experience different types of sexual harassment (visual, verbal and behavioral). Studies have shown that women's fear of public space limits their freedom and pleasure in public life and restricts their ability to benefit from opportunities and convenience (Mtani 2000). Research indicates that fear and safety are more related to subjective perceptions of neighborhood environment than objective measures of danger (Schafer, Huebner and Bynum, 2006). Therefore, many researches concentrate on the links between the physical characteristics of settings and perceived safety. Many scholars emphasize the complex interplay between social and physical realms and argue that adopting a simple set of design solutions is not enough (Booth, 1981; Merry, 1981; Taylor, Gottfredson and Brower, 1980). The new approach, stressing the socio- political constitution of fear has been highlighted by studies based on the critical tradition of social science, in particular feminist research (Koskela and Pain, 2000; Pain, 2000; Kyttä, 2011).

"Risk Taking", "Feeling Insecure", and "Fear of Crime" have different meanings among women like any other social science concept and the differences arise from the approach taken by researchers in this field. However, research conducted in this regard is classified into different categories and each of them has specific requirements.

The first category is related to sociological research that considers "Risk Taking", "Feeling Insecure", and "Fear of Crime" as dependent variables and link differences among the study population to other sociological variables based on these characteristics (Jagori Team, 2010; Loukaitou-Sideris, Liggett and Hiseki, 2002; Moser, 2004; Koskela and Pain, 2000; Buzan, 1983; De Jesus, Shelton and Emmons, 2010; Kagawa-Singer, Padilla and Ashing-Giwa, 2010).

The second category of the research is related to criminological research. In these studies, the focus is on violence and sexual harassment and the role that individual variables play in independent variables (such as genetic characteristics, personality characteristics, and psychological traits) in order to explain the distribution of social groups in 
terms of violence and sexual abuse (Buss, Larsen and Semmelroth, 1992).

The recent category of research deals with the environmental factors such as women's risk taking. In these studies, the physical conditions and characteristics of built environments establish defenseless spaces, because using these spaces is stressful and terrifying for women and it reduces their feeling of security.

Such studies relate to urban planning and design and consider the physical characteristics of urban spaces that affect people's risk taking, particularly women's. Studies conducted using this approach focus on variables like waiting in particular locations and crowded public transport/bus stations, lack of legibility, lack of safe and clean toilets for women in public spaces, and lack of lighting (Jagori Team, 2010); lack of gender indicators in urban planning and inappropriate sidewalks in poor areas, dim places and spaces (Loukaitou-Sideris, Liggett and Hiseki, 2002); dark paths and alleys, remote bus stations, public restrooms (Moser, 2004); shrubs and improper designed lawns, buildings with poor design and the general built environment (Koskela and Pain, 2000); poorly designed streets and houses (Pain, 2000); the physical characteristics of the neighborhood (Newman, 1972); lack of territoriality, natural surveillance, image (Newman, 1972); and other factors. Generally, the physical approach to women's fear of crime and risk taking is based on the fact that urban spaces are the main sources of fear of crime, insecurity, and risk taking, particularly women's. Despite the abovementioned, to date, there has been limited focus on how women and girls specifically experience safety and security at the neighborhood level (Collins, 2013). Today, Iranian women live in their neighborhoods, but they do not feel safe and comfortable. Some public spaces, particularly in certain areas of the Iranian cities are heavily dominated by men. Therefore, this study, in the context of a traditional neighborhood in Tehran, sought to understand the interrelationships between perceived safety by women and neighborhood planning and design. Given the possibility that men and women experienced safety for different reasons culturally and perception of security is a subjective area, it required a mixed method research to explore the association of individual predictors on perceptions of safety from contextual neighborhood contexts.

This study attempts to identify characteristics associated with women's feeling and perception of safety and security in urban areas. The findings of this paper can guide urban planners and designers to explore strategies to reduce environmental stresses and provide women with safe and peaceful neighborhoods. This might lead to the removal of restrictions on women to have active participation in the community and promote the well-being of the society.

\section{Methods}

\subsection{Field study}

A comprehensive plan is prepared for each of the 22 districts of Tehran by different groups of experts. This plan will be executed after complex processes of preparation and approval. According to studies on a comprehensive plan of District 10 of Tehran, a substantial portion of the Salsabil neighborhood can be considered as an urban deteriorated area. In addition, according to the studies carried out by Tehran's Renovation Organization (responsible for the preparation of urban renovation plan of the North Salsabil neighborhood), women of this area complained a lot about the social problems in this neighborhood and claimed that their presence in public spaces of this area is accompanied by fear of becoming victims of social deviation and crimes (Tehran's Renovation Organization,2012).

Based on the aforementioned explanations and considering the low level of women's safety in the Northern Salsabil neighborhood, this neighborhood was chosen as the case study area for this research. The total area is 97.3 hectares and its population is 36,178 based on the last census. $67 \%$ of this area is a deteriorated urban space.

The presence of the beggars and addicts, child labor, the presence of homeless women and prostitutes, conflict, theft, suicide, etc., are some of the most important social problems of this neighborhood. Moreover, the poor quality of physical buildings, lack of appropriate urban furniture, poor lighting of alleys and streets, the presence of notorious places and spaces, and uninhabited spaces are characteristics of the study area that directly or indirectly affect the feelings of safety and security of the residents, particularly women. (Tehran's Renovation Organization, 2012). 


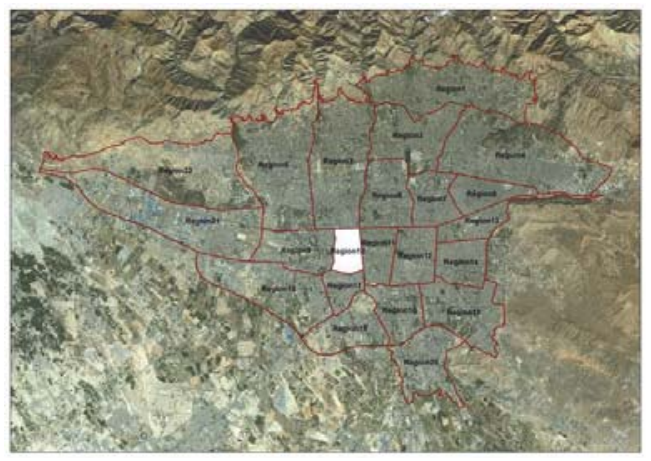

Figure 1: Region 10 Location in Tehran City (Authors 2015)

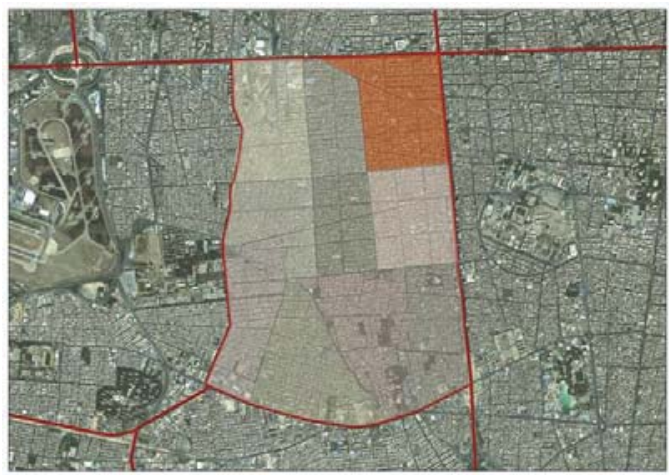

Figure 2: Case Study Location in Region 10 (Authors, 2015)

\subsection{2-2- Study design and sample}

In this study, a combination of quantitative and qualitative methods were used as a mixed research design to explore the factors associated with the perception of safety and security of women in the selected neighborhood. In the first instance, women's views about a safe neighborhood were sought by conducting semi-structured interviews (face to face) and they were asked the most important question: "What are the most affecting features on the neighborhood's security?" In addition, they were asked to explain the factors that caused fear, anxiety, and stress for them. They were also asked to clarify the most important safe and unsafe parts of the neighborhood and should provide reasons.

Table 1: Interview participants' characteristics (Total No.20)

\begin{tabular}{|c|c|c|}
\hline Age & (No.) & $(\%)$ \\
\hline $20-35$ & 7 & 35 \\
\hline $35-50$ & 7 & 35 \\
\hline $50-65$ & 6 & 30 \\
\hline Occupation & $($ No.) & $\%$ \\
\hline Householder & 7 & 35 \\
\hline Student/Collegian & 5 & 25 \\
\hline Jobholder & 5 & 25 \\
\hline Retired & 3 & 15 \\
\hline
\end{tabular}

Data saturation was continued to the sample size of 20, selected by snow-ball sampling, as Morse (1994) suggests that when the goal of a qualitative research is to understand the essence of an experience, researchers should use at least five participants (Morse 1994). 
Table 2: Qualitative Questions Asked from the Participants (Authors, 2015)

\begin{tabular}{|l|}
\hline 1. Why do you feel safe and secure in this neighborhood? \\
\hline 2. Why don't you feel safe and secure in this neighborhood? \\
\hline 3. Which part of the neighborhood is secure? Why? \\
\hline 4. Which part of the neighborhood is not secure? Why? \\
\hline 5. How can you feel more secure in the neighborhood? \\
\hline
\end{tabular}

Eligibility criteria included being a female 20-65 years old, living in the Salsabil neighborhood for at least 10 years and making use of neighborhood facilities and public spaces. Recruitment for the interviews was conducted within the North Salsabil neighborhood with the assistance of the North Salsabil civic building and Tehran's $10^{\text {th }}$ region municipality. All data were collected in summer 2015. Table 1 shows a summary of interviewed participants' characteristics.

Open-ended questions (Table 2) were used to gain feedback from the residents. Each respondent answered all the six open-ended questions on the semi-structured interview script. Each interview lasted for approximately 30 minutes. All interviews were recorded with the permission of the participants and subsequently transcribed. Written informed consent was also obtained from all study participants to assure that they will not be recognized in future. The authors discussed the importance of key concepts by reading the interview transcripts and taking notes on phrases and concepts in the content analysis. Research notes were first written down individually by the researchers with the aim of identifying broad thematic elements and discrete meaning units for coding purposes. Words, phrases, sentences or paragraphs were read over and over in the transcript text as the process of analyzing the transcripts progressed. Researchers discussed their individual perceptions of the discrete meaning units study sections to further refine the concepts into logical broad categories, more specific subcategories, and conceptual model of the study. The goal in this stage was a triangulation to ensure trustworthiness. General thematic categories and subcategories evolved and were collectively represented by general categories with 11 main themes, using content analysis in the qualitative phase (Table 3) to convey the interrelated connections in the conceptual model of the research across the three broad themes: person, community, and the built environment (Table 4).

Table 3: General Thematic Categories and Themes (Authors, 2015)

\begin{tabular}{|l|l|}
\hline General Categories & Themes \\
\hline Person & - Age \\
& - Marriage \\
& -Coating Type (Islamic Dressing) \\
& -Socioeconomic Status \\
\hline Community & -Delinquency, Vandalism and Robbery \\
& -Surveillance in Neighborhood Space \\
& - Social Capital \\
& - Culture \\
\hline Built environment & - Territoriality \\
& - Livability and vitality \\
& -Urban pattern (organic/ grid) \\
\hline
\end{tabular}

Table 4: The Research Conceptual Model (Authors, 2015)

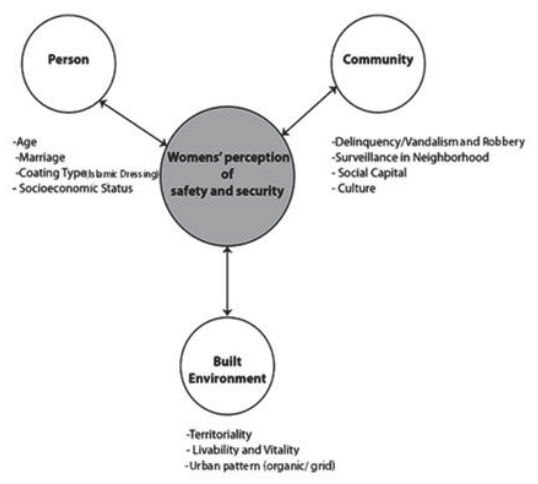


Table 5: Respondents' characteristics (Total No.200)

\begin{tabular}{|c|c|c|}
\hline Age & (No.) & $(\%)$ \\
\hline $20-35$ & 70 & 35 \\
\hline $35-50$ & 78 & 39 \\
\hline $50-65$ & 52 & 26 \\
\hline Occupation & (No.) & $\%$ \\
\hline Householder & 10 & 50 \\
\hline Student/Collegian & 36 & 18 \\
\hline Jobholder & 44 & 22 \\
\hline Retired & 20 & 10 \\
\hline
\end{tabular}

After determining the conceptual model, quantitative methods were used at a later stage, because of the limited statistical population (respondents) and to avoid hasty and incorrect generalizations in assessing conceptual model. For this reason, various aspects of the conceptual model were formulated into statements and questions, and a questionnaire consisting of 4 main parts and 22 items was developed. Likert scale (Very high (score=5), high (score=4), moderate (score=3), low (score=2), and very low (score=1)) was used to answer the questions. Cronbach's alpha was used to prove the reliability of the questionnaire. In order to ensure the validity of the questionnaire, five experts were asked to assess the questionnaire items. The results revealed that the content validity ratio was more than 0.99 for all of the 22 items of the questionnaire which is an acceptable ratio. The first part of the questionnaire is related to personal characteristics of respondents. The second part is planned to determine the relationship between women's personal characteristics (age, marital status, veil and Socioeconomic Status) and their perception of safety and security (Cronbach's alpha: 0.776 ). The third part focuses on the community characteristics and aims to measure the relationship between them and women's perception of safety and security, including 7 statements (Cronbach's alpha: 0.851). The fourth part of the questionnaire includes 7 statements that made it becomes possible to determine environmental characteristics contributing to women's perception of safety and security and their level of association (Cronbach's alpha: 0.889). The sample size was set as 200 on Cochran's formula in the neighborhood.

Recruitment for the questionnaires was conducted with the assistance of the North Salsabil health center and Tehran's 10th region municipality. Eligibility criteria included 20-65 aged women, living in North Salsabil, making use of North Salsabil health center services. Table 5 shows the age and occupation of the respondents. Information obtained through the questionnaire was analyzed using SPSS19 software and the frequency of each of the factors contributing to women's perceptions of safety and security in the Northern Salsabil neighborhood was calculated.

\section{Results}

In this study, the residents of the selected neighborhood were asked to answer the questions raised in the form of semistructured in-depth interviews. The conceptual model of this research was formed by women's responses to the following: questions relating to the safe and unsafe areas of the neighborhood, the most important factors affecting the safety and security of women in the area, and factors causing fear and anxiety. According to this model, the variables affecting the perception of safety and security in urban areas at the neighborhood scale can be divided into three main dimensions: characteristics of the individual, the local community, and the built environment.

A questionnaire was prepared after providing the conceptual model. In order to test the generalizability of the questionnaire, 200 women were asked about the factors affecting their perception of security. The following tables show the statistical results of the questionnaire on each of the three factors. Table 6 shows the spectrum of perception of safety and security in the neighborhood. 
Table 6: Measures Affecting Women's Perception of Neighborhood Safety and Security (Authors, 2015)

\begin{tabular}{|c|c|c|}
\hline Individual Characteristics & Local Community Characteristics & Built Environment Characteristics \\
\hline $\begin{array}{ll}\text { - } & \text { Age } \\
\text { - } & \text { Marital Status } \\
\text { - } & \text { Coating Type (Islamic } \\
\text { Dressing) } \\
\text { - } \\
\text { Socioeconomic Status } \\
\text { of the Person }\end{array}$ & 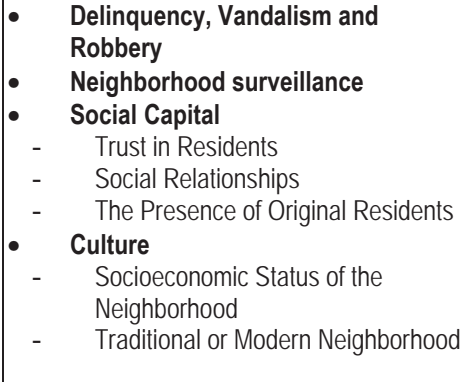 & \begin{tabular}{|cc}
- & Territoriality \\
- & Designed Spaces \\
- & Lost Spaces \\
- & Livability/Vitality \\
- & Lighting, Safe Accessibility and Safe Design of \\
- & Be Intersections \\
- & Urbanioral Settings(Milieus) \\
- & Vacant and Abandoned Lands) \\
& Good Pavement, Sidewalks and Pathways and \\
Types of Pollutants \\
Urban pattern (organic/ grid)
\end{tabular} \\
\hline
\end{tabular}

\subsection{Personal characteristics analyses}

Table 7 presents the statistical analysis of the personal characteristics contributing to the women's feeling and perception of safety and security. In response to the question "Which components of individual characteristics are important in women's perception of the neighborhood's safety and security?", the participants introduced age as the most important component (total score: 774). Of all the respondents, $68 \%$ suggested that these factors affect safety and security very much, $70 \%$ suggested the effect of the factors was very high, 35\% suggested the effect of the factors was average and $27 \%$ suggested the effect of the factors was low or very low. Based on the results of the questionnaire, the most vulnerable age group was the 15 to 30 age group who felt the need for more support, followed by 30 to 45 years age group and the group older than 45 years.

According to women, attire and Islamic dressing is very important in their feeling of safety (total score of 712). In this study, $32 \%$ of the women mentioned the importance of the veil as very high, $27 \%$ as high, $17 \%$ as moderate, $12.5 \%$ as low and only $9.5 \%$ mentioned was very low. Marital status with a score of 683 was introduced as the third important individual feature in perception of security as $22 \%$ of the women considered the effect of this variable as very high, $28.5 \%$ as high, $24.5 \%$ as moderate, $19 \%$ as low and $12 \%$ considered it to be very low. In addressing the marriage issue, most women considered being single as the reason for higher vulnerability in the neighborhood.

Finally, SES (total score of 610 ) was set at the fourth rank; $15 \%$ of the women reported the effect of this variable to be very high on security perception, $25 \%$ as high, $25 \%$ as moderate, $20 \%$ as low and $15 \%$ reported it as very low. People with higher socio-economic level (employment status, income) felt less safe and secure in the neighborhood.

Table 7: Statistical Analysis of the Personal Characteristics (F=Frequency, P=percentage) (Authors, 2015)

\begin{tabular}{|c|c|c|c|c|c|c|c|c|c|c|c|c|c|c|}
\hline \multirow{2}{*}{ Criteria } & \multicolumn{2}{|c|}{ Very High } & \multicolumn{2}{|c|}{ High } & \multicolumn{2}{|c|}{ Moderate } & \multicolumn{2}{|c|}{ Low } & \multicolumn{2}{|c|}{ Very Low } & \multicolumn{2}{|c|}{ Not Answered } & \multicolumn{2}{|c|}{ Total } \\
\hline & No. & $\%$ & No. & $\%$ & No. & $\%$ & No. & $\%$ & No. & $\%$ & No. & $\%$ & No. & $\%$ \\
\hline Marriage & 44 & 22 & 57 & 28.5 & 49 & 24.5 & 38 & 19 & 12 & 6 & 0 & 0 & 200 & 100 \\
\hline Age & 68 & 34 & 79 & 35 & 35 & 17.5 & 22 & 11 & 5 & 2.5 & 0 & 0 & 200 & 100 \\
\hline Coating type(Islamic dressing) & 66 & 32.5 & 54 & 27 & 34 & 17 & 25 & 12.5 & 19 & 9.5 & 3 & 1.5 & 200 & 100 \\
\hline Socioeconomic Status & 30 & 15 & 50 & 25 & 50 & 25 & 40 & 20 & 30 & 15 & 0 & 0 & 200 & 100 \\
\hline
\end{tabular}

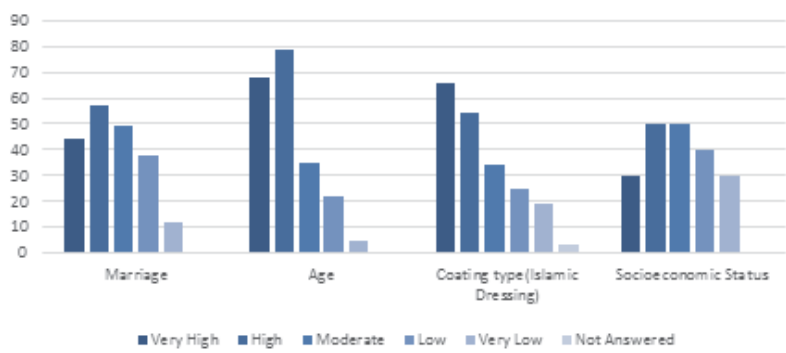

Chart 1: Statistical Analysis of the Personal Characteristics (Authors, 2015) 


\subsection{Community features analyses}

In response to the question "Which component of the community factor has the greatest effect on safety and security perception?", the majority of the respondents mentioned delinquency, vandalism and robbery as the most important factor in the neighborhood (total score of 700). Of all the respondents, $27.5 \%$ have considered it to be very high, 30\% as high, $19 \%$ as moderate, and $21 \%$ have considered it to be low or very low.

At the second level, neighborhood surveillance (total score of 681) was mentioned as the most important variable of the community factor; thus, $31.5 \%$ of women mentioned the importance of this component in security perception as very high, $23 \%$ as high, $15 \%$ as moderate, $16.5 \%$ as low and $13 \%$ as very low.

After neighborhood surveillance, social capital was considered in the form of trust in people, social relationships and the presence of local residents in the neighborhood provided security (total score of 557). 14\% of the respondents considered the importance of this component to be very high, $21 \%$ as high, $20 \%$ as moderate, $20 \%$ as low, and $24.5 \%$ as very low.

Finally, the respondents focused on the culture items (with measures of neighborhood socio- economic level and the traditional/modern status of neighborhoods), with the total score of $478.10 \%$ mentioned the effect of this component to be very high, $24.5 \%$ as high, $20.5 \%$ as moderate, $24 \%$ as low, and $20 \%$ as too low.

Table 8 shows how community features influence women's feeling and perception of safety and security in the neighborhood.

Table 8: Statistical Analysis of the Community Characteristics (F=Frequency , P=percentage) (Authors, 2015)

\begin{tabular}{|c|c|c|c|c|c|c|c|c|c|c|c|c|c|c|}
\hline \multirow{2}{*}{ Criteria } & \multicolumn{2}{|c|}{ Very High } & \multicolumn{2}{|c|}{ High } & \multicolumn{2}{|c|}{\begin{tabular}{|l|} 
Moderate \\
\end{tabular}} & \multicolumn{2}{|c|}{ Low } & \multicolumn{2}{|c|}{ Very Low } & \multicolumn{2}{|c|}{ Not Answered } & \multicolumn{2}{|c|}{ Total } \\
\hline & No. & $\%$ & No. & $\%$ & No. & $\%$ & No. & $\%$ & No. & $\%$ & No. & $\%$ & No. & $\%$ \\
\hline Neighborhood surveillance & 63 & 31.5 & 46 & 23 & 30 & 15 & 33 & 16.5 & 26 & 13 & 2 & 1 & 200 & 100 \\
\hline Socia & 28 & 14 & 42 & 21 & 40 & 20 & 40 & 20 & 49 & 24.5 & 1 & 0.5 & 200 & 100 \\
\hline & 20 & 10 & 49 & 24.5 & 41 & 20.5 & 48 & 24 & 40 & 20 & 2 & 1 & \begin{tabular}{|l|l|}
200 \\
\end{tabular} & 100 \\
\hline Delinquency, Vandalism and Robbery & 55 & 27.5 & 60 & 30 & 38 & 19 & 28 & 14 & 15 & 7.5 & 4 & 2 & 200 & 100 \\
\hline
\end{tabular}

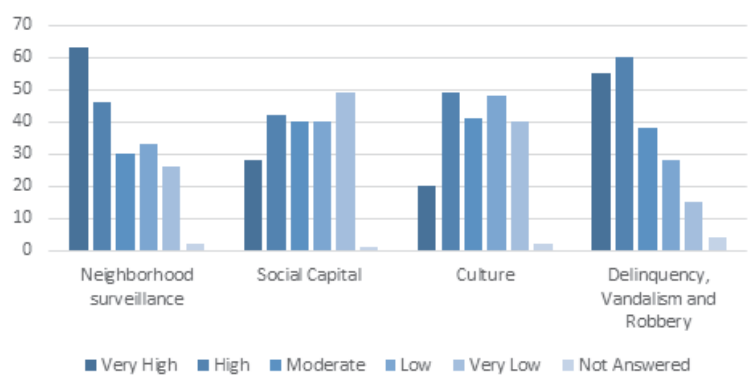

Chart 2: Statistical Analysis of Community Characteristics (Authors, 2015)

\subsection{Built environment characteristics analyses}

Table 9 shows the statistical analysis of the built environment characteristics affecting women's feeling and perception of safety and security in the neighborhood.

As shown in Table 9, in the first step, the most important components affecting safety and security perception of the built environment (man-made environment) were the territoriality of urban spaces (public, semi-public, semi-private, and private spaces), and lack of lost space (total score of 741); $45 \%$ of the respondents mentioned the effect of this variable as very high, $24 \%$ as high, $4.5 \%$ as moderate, $9.5 \%$ as low, and $17 \%$ as very low.

In the second level, the livability and vitality of the neighborhood were measured by three variables. The neighborhood lighting (urban landscape), access to all areas of the neighborhood (sufficient permeability both physical and visual), and geometric and safe design of the intersections and routes (total score of 661) formed the first level of the feature of the built environment livability. The presence of behavioral settings in the built environment involved social interactions that are very important in affecting women's perception of safety and security with a total score of 652 . 
According to women, the presence of demolished buildings, wasteland, and degraded and abandoned lands (total score of 595) were set in the third level of variables defining built environment livability. Also, pavements, sidewalks, and environmental pollution (total score of 583) affected women's perception of neighborhood safety and security.

Neighborhood pattern with the total score of $\mathbf{5 5 6}$ was considered in the third level of components affecting the safety and security of the built environment; therefore, if the urban texture is grid, women perceive the environment as safer if the urban texture is more organic and unordered, women perceive it as unsafe. Of the respondents, 14\% mentioned this effect to be very high, $17.5 \%$ as high, $23.5 \%$ as moderate, and $45 \%$ mentioned very low or low.

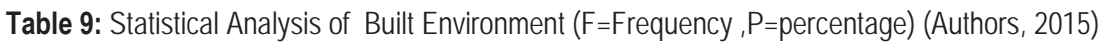

\begin{tabular}{|c|c|c|c|c|c|c|c|c|c|c|c|c|c|c|c|}
\hline \multirow{2}{*}{\multicolumn{2}{|c|}{ Criteria }} & \multicolumn{2}{|c|}{ Very High } & \multicolumn{2}{|c|}{ High } & \multicolumn{2}{|c|}{ Moderate } & \multicolumn{2}{|c|}{ Low } & \multicolumn{2}{|c|}{ Very Low } & \multicolumn{2}{|c|}{ Not Answered } & \multicolumn{2}{|c|}{ Total } \\
\hline & & No. & $\%$ & No. & $\%$ & No. & $\%$ & No. & $\%$ & No. & $\%$ & No. & $\%$ & No. & $\%$ \\
\hline \multicolumn{2}{|c|}{$\begin{array}{l}\text { Territoriality : } \\
\text { Designed Spaces/Lost Spaces }\end{array}$} & 90 & 45 & 48 & 24 & 9 & 4.5 & 19 & 9.5 & 34 & 17 & 0 & 0 & 200 & \\
\hline \multirow{4}{*}{ 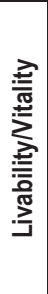 } & \begin{tabular}{|l}
-Behavioral Settings \\
(Milieus)
\end{tabular} & 52 & 26 & 55 & 28 & 23 & 11.5 & 33 & 16.5 & 37 & 18.5 & 0 & 0 & 200 & 100 \\
\hline & $\begin{array}{l}\text {-Urban Decay } \\
\text {-Old Fabric / Vacant Lands }\end{array}$ & 35 & 17.5 & 58 & 29 & 26 & 13 & 32 & 16 & 47 & 23.5 & 2 & 1 & 200 & 100 \\
\hline & \begin{tabular}{|l}
-Lighting, \\
- Safe Accessibility \\
-Safe Intersections
\end{tabular} & 48 & 24 & 43 & 22 & 54 & 27 & 32 & 16 & 23 & 11.5 & 0 & 0 & 200 & 100 \\
\hline & $\begin{array}{l}\text { - Pavement } \\
\text {-Types of Pollutants }\end{array}$ & 35 & 17.5 & 40 & 20 & 45 & 22.5 & 35 & 17.5 & 43 & 21.5 & 2 & 1 & 200 & 100 \\
\hline \multicolumn{2}{|r|}{ Urban pattern (organic/ grid) } & 28 & 14 & 35 & 18 & 47 & 23.5 & 45 & 22.5 & 45 & 22.5 & 0 & 0 & 200 & 100 \\
\hline
\end{tabular}

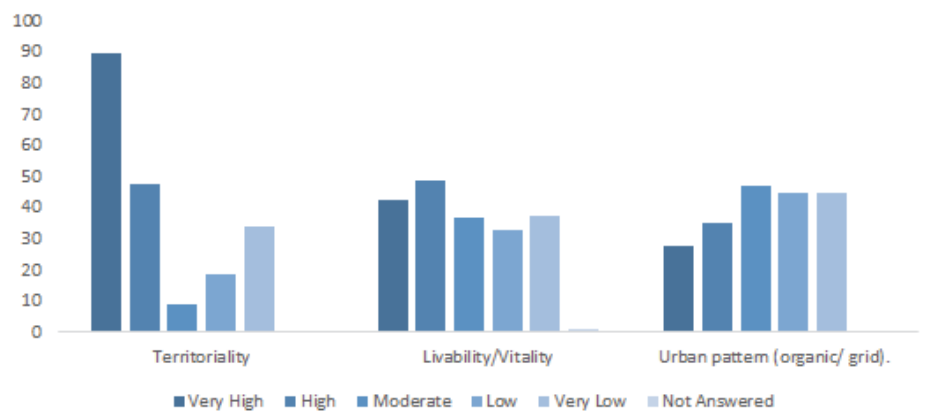

Chart 3: Statistical Analysis of Built Environment (Authors, 2015)

\section{Discussion}

This research is an attempt to identify the factors that affect women's perception of safety and security in the neighborhood; in other words, this study aims to figure out how women experience safety and security in their neighborhood in both subjective and objective attributes. It has long been recognized that the built environment alone will not create safer communities (Booth,1981; Merry, 1981;Taylor, Gottfredson and Brower, 1980).

Personal characteristics, community characteristics, and the built environment were identified as three main factors based on women's own narratives of their perception of safety and security in Tehran. The findings of our study are in line with other similar studies.

For example, Jagori and colleagues' report revealed that three factors including physical characteristics (lighting, signage, visibility, isolation, escape routes, maintenance), social organizations and policing (social surveillance/control) and usage of spaces were deemed effective in women's perception of safety and security in Delhi (Jagori Team, 2010).

It seems that the similarity between the identified criteria in both researches can be attributed to social and cultural roots in eastern countries, such as Iran and India. This issue should be interpreted, because safety and security are 
completely dependent on culture. For example, in another study carried out in Iran, four fundamental characteristics including SES, control characteristics (surveillance bodies such as the police), social characteristics (sense of community) and physical characteristics were considered effective in the fear of crime (Alikhah and Najibi Rabiee, 2006).

Among the personal factors, the age of women is regarded as the most important item in the perception of safety and security. Most of the requests for support were made by women between 15 and 30 years old, this could be attributed to the sexual harassment concerns of women. Our findings showed that veil is the second important factor that elevates the safety and security of women in the neighborhood. However, to the best of our knowledge, no other similar studies have found the literature to consider this issue as this would be an important subject in Islamic cultures and communities. Some people in the community of Iran believe that women who have Islamic dresses are safer against sexual harassment (verbal and visual).

Marital status also plays an important role in the women's perception of safety, as introduced by other studies (Keane, 1998; Ferraro, 1995). One of the possible explanations for feeling safety for married women might be their confidence after marriage to encounter with contra sex would be increased. Moreover, single women feel more aloneness against violence in urban spaces.

Finally, women who are in lower socioeconomic conditions feel less safe in these residential environments. This can be related to long working hours for women and their continuous working until midnight in order to earn a living. In other studies, it is also proven that SES of people increases their fear of crime (Pantazis, 2000).

The second factor in this research that affected women's perceptions of safety and security is the characteristics of the local community. In this connection, delinquency, vandalism, and robbery were the most important factors, which result in women's experiences of safety in their neighborhood. The absence of delinquency, theft, and crime (drugs) are among the factors that increased women's perception of safety at different times of the day and night. Other studies support our findings indicating that delinquency and crime are important security factors in the neighborhood (Foster, Giles-Corti and Knuiman, 2011; Caughy, O'Campo and Patterson, 2001; Perkins, Meeks and Taylor, 1992).

Social control through surveillance of neighborhoods, permanent presence of residents, and eye on the street in the neighborhood are among the other significant factors that have contributed to women's experience of safety and their presence in all places at all times in the whole neighborhood. Sampson and Graif showed that social control is one of the important elements in making neighborhoods safer ( Sampson and Graif, 2009). The next factor is social capital and social cohesion that provide a safer and more secure neighborhood for women by developing trust and helpfulness among the residents. The importance of social capital in higher perceptions of safety also depends on the presence of original and local residents, the extent of familiarity, and trust between them. Finally, the SES in the neighborhood is also one of the considerable factors in women's perception of safety. In a high socioeconomic situation, community people behave in a respectful and good manner.

The third significant and effective dimension in women's perception of safety and security is the built environment. Some of reports have highlighted neighborhood physical attributes in incivility items pertaining to decayed and run-down buildings, abandoned buildings, vacant lots, debris in the streets, graffiti, and poor lighting (Pitner, ManSoo and Brown, 2012).

In this study, having a hierarchy of spaces has the greatest impact on providing the ground for better social control. Territoriality is one of the substantial characteristics of environment that has been proposed by Crime Prevention through Environmental Design (CPTED) to have more control by local residents increasing safety (Newman, 1972; Brown, Perkins and Brown, 2004; Cozens, Saville and Hillier, 2005). Second delinquency in the neighborhood can be related to the abandoned lands and the possibility of escape and concealment in the neighborhood. Factors such as the neighborhood lighting (urban landscape), access to all areas of the neighborhood (sufficient permeability both physical and visual) and geometric and safe design of the intersections and routes encourage people into the public realm and facilitate pedestrian movement throughout the neighborhood to interact more (Pitner, ManSoo and Brown, 2012). If the neighborhood also has mixed-use facilities to invite women into the public realm, and well-connected streets to facilitate walking; then, more women may walk in their neighborhoods. It follows that the neighborhood may help alleviate fear of crime, because residents feel safer when more people circulate through the neighborhood and supports the assertion that 'eyes on the street' enhance perceptions of safety (Foster, Giles-Corti and Knuiman, 2011b). The role of these physical factors in creation of safe neighborhoods has also been emphasized in other similar studies, such as lighting, signage, visibility, isolation, escape routes, and maintenance (Jagori Team, 2010).

In addition, the presence of livable neighborhood increases social interactions, although, demolished buildings, wasteland and degraded and abandoned lands decrease the presence of women in neighborhoods' urban spaces as a result of refuse pertaining to decayed and run-down buildings, abandoned buildings, vacant lots, debris in the streets, graffiti, and poor lighting increasing the possibility of hiding and disturbing (Pitner, ManSoo and Brown, 2012). 
Besides, the quality of pollution in neighborhood as well as density and contaminations are among other factors that affect the presence of women in the neighborhood. For instance, the quality of pathways causes the improvement of the aesthetic presentation of the street, making it safe for walking, without fear of falling down; or influencing perceptions of safety either directly (through potential for surveillance and maintenance standards) or indirectly (through their capacity to discourage expressions of antisocial behavior). Indeed, there is evidence to support these ( Foster, Giles-Corti and Knuiman, 2011b).

Previous studies highlighted a direct association between incivilities and physical activity (which was not mediated by perceived safety) (Miles, 2008), whereas another identified perceived safety as a mediator of the association between objective physical incivilities and total physical activity (King, 2008; Foster, Giles-Corti and Knuiman, 2011b).

The existence of grid patterns instead of organic patterns in the neighborhood is another item (mentioned by women themselves) that affects women's perception of safety and security. The reason for this can be attributed to perception of disorder and lack of legibility in the organic and creation of unknown spaces that has led to increased fear in women. Since the grid pattern creates a clearer mental image in people. This has also been confirmed in previous studies as residents in neighborhoods with more perceived disorder have higher levels of fear (Ross and Jang, 2000), and higher status neighborhoods are associated with less disorder and crime (McCord, et al. 2007).

The most important limitations in the way of this study are the following:

- The study is limited to an old neighborhood which is poor in SES with low level of women's safety and security upon Tehran's municipality report.

- Conducting formal studies about sexual harassments is not possible in traditional society of Iran.

- Need to set up a tool for measuring perception of safety and security in Tehran's neighborhoods

- Lack of efficient databases, for example GIS databases, to assess other neighborhood physical attributes.

\section{Conclusion}

This study tested the factors that associate the safety and security perceptions of women in a neighborhood based on the necessity and importance of providing safe and comfortable presence for women in residential areas. In this regard, a combination of qualitative and quantitative methods was used and its generalizability was examined after developing the conceptual model. According to the results, the three factors of individual characteristics, local community features, and built environment were the most influential factors defining the concept of women security in a neighborhood. In this study, it was found that as compared to the other two factors, the role of the built environment was more prominent in urban design and planning and there is possibility of physical intervention in this area more than the other two factors. The results revealed that women's perception of safety and security in a neighborhood includes both procedural and substantive dimensions; individual components and local community are important in procedural dimensions. Increased social capital in neighborhoods and improved social interactions, increased quality of life, improved socio-economic conditions of neighborhood, preserved the local context of inhabitants, promote and preserved the traditions and culture of the neighborhood are effective factors of procedural dimensions that help women feel safer and more secure.

The findings of this study revealed that creating a territoriality (spatial hierarchy of the neighborhood) can improve the perception of safety and security, that is, transition from public to semi-public, semi-private and private spaces of housing units in a neighborhood. There are some measures that make the environment more acceptable and comfortable for women in terms of safety and security, and they are as follows: improving the vitality of the neighborhoods' urban spaces by building shopping centers and main streets, landscaping, lighting and providing suitable pavement and proper slope of passages with designed geometric intersections (reduced accident rate), reducing the amount of wasteland and degraded/abandoned lands and reducing the pollutants in the streets and neighborhood centers. The change in the organic pattern of the neighborhood towards grid pattern where the possibility of criminals hiding and escaping is reduced is the general preference of women. It can be concluded that making improvements in the safety and security of women in a neighborhood will be possible through conducting physical interventions primarily on neighborhood livability, which is within the expertise of architects and urban designers. However, the greatest effect on women's feeling of safety and security is associated with personal and social features of the neighborhood environment, which can be boosted by improving the quality of life. This paper has come up with a set of recommendations on how women safety and security can be further improved in the capital city and make it a women safe in their neighborhoods. The recommendations focus on the following:

- enhancing neighborhood centers by mixed-use and diversity to build more livable neighborhood

- improving the quality of built environment to attract more women to be present in neighborhoods 
- Introduction of women friendly public transport systems

- Fostering support from the general public by engaging them effectively across various

- Levels and sections of the society including educational institutions, cultural activities to make women more confident.

- Urban design and planning can make an impact on women's experience of safety. For example,

- Use of spaces for a diversity of purposes is more conducive to the production of safety. The concept of 'eyes on the street' is the only solution to lack of safety, rather than getting people off the streets.

\section{Acknowledgements}

The authors thank Prof. Jahanshah Pakzad and Dr Hamid R Baradaran for guiding this study. The authors would also like to thank female residents of the Salsabil neighborhood who helped in improving this research. Special thanks of Mrs. Monika Nazemzadeh and David Chandler, MA for translations.

\section{References}

Alikhah, Fardin, and Maryam. Najibi Rabiee. 2006. "Women and Fear of Crime in Urban Alikhah, Fardin, and Maryam. Najibi Rabiee. ( 2006). Women and Fear of Crime in Urban Spaces. Social Welfare, 22:109-131.

Booth, Alan. 1981.. The built environment as a crime deterrent. Criminology, Volume 18, Issue 4, 557-570.

Buss, David. M., Randy J. Larson, Drew Westen, and Jennifer Semmelroth.(1992). Sex Differences in Jealousy: Evolution, Physiology and Psychology. American Psychological Society, 4, 251-255.

Buzan, Barry. (1983). People, states, and fear: the national security problem in international relations, Chapel Hill: University of North Carolina press.

Brown , Barbara. B., Douglas D.Perkins and Graham Brown. (2004). Crime, new housing, and housing incivilities in a first-ring suburb: multilevel relationships across time. Housing Policy Debate,15(2), 301-345.

Caughy, Margaret O'Brien, Patricia O'Campo and Jacqueline Patterson. (2001). A brief observational measure for urban neighborhoods . Health \& Place, 7(3): 225-236.

Collins, Robin. (2013). Women and Girls' Eyes on the Neighbourhood: Feeling Safe in Public Space. (PQCHC), Pinecrest-Queensway Community Health; (CAWI), City for All Women Initiative.

Cozens, Michael .P., Gerg Saville and David Hillier. (2005). crime prevention through environmental design (CPTED):A review and modern bibliography. Property Management, 23(5), 328-356.

De. Jesus Maria, Puleo M. E., Shelton ,R. C., and M. Emmons .K. (2010). Associations between Preceived Social Environment and Neighborhood Safety: Health Implications. Health \& Place, 16(5) :1007-1013.

Ferraro, Kenneth F .(1995). Fear of Crime: Interpreting Victimization Risk. New York: SUNY Press.

Foster, Sara, Billie Giles-Corti, and Matthew Knuiman. (2011a). Creating safe walkable streetscapes: Does house design and upkeep discourage. Environmental Psychology, 1.79-88.

Foster, Sara, Billie Giles-Corti, and Matthew Knuiman .(2011b). Neighbourhood design and fear of crime: A social-ecological examination of the correlates of residents' fear in new suburban housing developments. Health \& Place, 16(6):1156-1165.

Jagori Team. (2010). Underestanding Women's Safety: Towards a Gender Inclusive City. research findings. Delhi: Jagori.

Kagawa-Singer Marjorie, Geraldine V. Padilla and Kimlin Ashing-Giwa. (2010). Health-Related Quality of Life and Culture. Seminars in Oncology Nursing, 26(1): 59-67.

Keane, Carl. (1998). Evaluating the influence of fear of crime as an environmental mobility restriction on women's routine activities. Environment and Behavior, 30: 60-74.

King, D. (2008). Neighborhood and individual factors in activity in older adults: results from the neighborhood and senior health study. Aging \& Physical Activity, 16(2):144-170.

Koskela, Hille, and Rachel Pain. (2000). Revisiting Fear and Place: Women's Fear of Attack and the Built Environment. Geoforum, 31(2): 269-280.

Kyttä, Marketta. (2011). SoftGIS methods in planning evaluation. In Evaluation for Participation and Sustainability in Planning, edited by Angela Hull, E. R. Alexander and Johan Woltjer, 334-354. London and New York: Routledge.

Loukaitou-Sideris, Anastasia., Robin Liggett and Hiroyuki Iseki. (2002). The geography of transit crime: Documentation and Evaluation of Crime Incidence on and around the Green Line Stations in Los Angeles.Planning Education and Research, 2, 135-151.

McCord, Eric S, Jerry H. Ratcliffe, R. Marie Garcia and Ralph B. Taylor. (2007). Nonresidential crime attractors and generators elevate perceived neighborhood crime and incivilities. Research in Crime, 3, 95-320.

Merry, Sally. E. (1981). Defensible space undefended: social factors in crime control. Urban Affairs Quarterl, 16(4), 397-422.

Miles, Rebecca. (2008). Neighborhood Disorder, Perceived Safety, and Readiness to Encourage Use of Local Playgrounds. American Journal of Preventive Medicine, 34(4), 275-281.

Morse, Janice .M. (1994). Designing funded qualitative research. In Norman K. Denzin \& Yvonna S. Lincoln (Eds.), Handbook of qualitative research (2nd ed.). Thousand Oaks, CA: Sage, 35-220. 
Moser, Caroline O N. (2004). Urban Violence and Insecurity: An Introductory Roadmap. Environment and urbanization, 2: 3-16.

Mtani, Anna .(2000). Violence and women's city: meeting places and places of interaction, experience of Dar es Salaam. paper delivered at the Naples Conference.

Nasar, Jack. L. and Kym M. Jones . (1997). Landscapes of Fear and Stress. Environment and Behavior,29(3), 291-323.

Newman, Oscar. (1972). Defensible Space: Crime Prevention through Urben Design. New York: Macmillian.

Pain, Rachel. (2000). "Place, social relations and the fear of crime: a review. Progress in Human Geography ".Progress in Human Geography, 3, 365-387.

Pantazis, Christina. (2000). Fear of Crime', Vulnerability and Poverty. Evidence from the British Crime Survey. British Journal of Criminology, 40(3), 414-436.

Perkins, Douglas, John W. Meeks and Ralph B. Taylor. (1992). The physical environment of street blocks and resident perceptions of crime and disorder: implications for theory and measurement. Journal of Environmental Psychology , 12(1) , 21-34.

Pitner, Ronald. O., Mansoo Yu and Edna Brown. (2012). Making neighborhoods safer: Examining predictors of residents' concerns about neighborhood safety. Journal of Environmental Psychology,32(1), 43-49.

Ross, Catherine .E and Sung Joon Jang. (2000). Neighborhood Disorder, Fear, and Mistrust: The Buffering Role of Social Ties with Neighbors. American Journal of Community Psychology, 28(4), 401-420.

Sampson Robert J. and Corina Graif. (2009). Neighborhood social capital as differential social organization: Resident and leadership dimensions. American Behavioral Scientist ,52(11), 1579-1605.

Schafer, Joseph A., Beth M. Huebner and Timothy Bynum . (2006). Fear of crime and criminal victimization: Gender-based contrasts. Journal of Criminal Justice, $34(3): 285-301$.

Taylor, Ralph. B., Stephen D.Gottfredson, and Sidney Brower (1980). The defensibility of defensible space: A critical review and a synthetic framework for future research in Understanding crime. Current, edited by Travis Hirschi and Michael Gottredson, 53-71. Beverly Hills: Sage publications.

Tehran's Renovation Organization. (2012). Urban Renovation Plan of Salsabil's Neighorhood's deteriorated areas. Project Report, Tehran: Tehran's Municipality. 